\title{
Missed mesenteric injury in blunt abdominal trauma: A potentially lethal error
}

\author{
Victor Kong, John Burce, George Oosthuizen, Grant Laing, \\ Damian Clarke
}

\begin{abstract}
Introduction: Mesenteric injury following blunt abdominal trauma is uncommon. Delay in diagnosis is unfortunately common due to its subtle clinical presentation and is associated with significant morbidity and mortality. Case Report: We present a case of a 35-year-old male who was discharged home with a missed mesenteric injury. The initial computed tomography scan of the abdomen and pelvis was normal. He re-presented acutely with small bowel infarction. Conclusion: Although computed tomography is the current gold standard of investigation, a very small proportion of injuries that require surgical intervention will invariably be missed. This case highlights several pitfalls in evaluation of these patients. Clinicians must always be vigilant of such injury despite normal radiological studies. A sufficient period of observation post injury should be considered in circumstances where
\end{abstract}

Victor Kong ${ }^{1}$, John Burce ${ }^{2}$, George Oosthuizen³, Grant Laing $^{2}$, Damian Clarke ${ }^{3}$

Affiliations: ${ }^{1} \mathrm{MBChB}$, Surgical Registrar, Pietermaritzburg Metropolitan Trauma Service, Department of Surgery, Edendale Hospital, Pietermaritzburg, KwaZulu Natal, South Africa; ${ }^{2} \mathrm{FCS}(\mathrm{SA})$, Consultant Trauma Surgeon, Pietermaritzburg Metropolitan Trauma Service, Department of Surgery, Edendale Hospital, Pietermaritzburg, KwaZulu Natal, South Africa; ${ }^{3} \mathrm{FCS}(\mathrm{SA})$, Senior Consultant Trauma Surgeon, Pietermaritzburg Metropolitan Trauma Service, Department of Surgery, Edendale Hospital, Pietermaritzburg, KwaZulu Natal, South Africa .

Corresponding Author: Victor Yeewai Kong, Department of Surgery, Edendale Hospital, Private Bag X509, Plessislaer Pietermaritzburg, 3216, KwaZulu Natal, South Africa; Ph: +27(0)797411036; Fax: +27(0)333954094; Email: victorywkong@yahoo.com

Received: 21 November 2012

Accepted: 11 December 2012

Published: 01 July 2013 the possibility of mesenteric injury is increased. Such circumstances include lack of seat belt usage, high-speed impact and deceleration injury.

Keywords: Mesentery, Trauma, Missed injuries, Blunt

$$
* * * * * * * * *
$$

Kong V, Burce J, Oosthuizen G, Laing G, Clarke D. Missed mesenteric injury in blunt abdominal trauma: A potentially lethal error. International Journal of Case Reports and Images 2013;4(7):376-379.

$$
* * * * * * * * *
$$

doi:10.5348/ijcri-2013-07-335-9

\section{INTRODUCTION}

Mesenteric injury following blunt abdominal trauma is uncommon and the diagnosis is particularly difficult as clinical presentation is often subtle $[1,2]$. Although computed tomography (CT) scan of the abdomen is the method of choice for the evaluation of these patients, in a very small proportion of cases, the diagnosis can invariably be missed and could potentially lead to disastrous outcome [3]. We present a case of massive mesenteric disruption complicated by small bowel infarction in a 35-year-old male who had subtle clinical signs and normal CT scan of the abdomen on initial evaluation.

\section{CASE REPORT}

A 35-year-old male was presented to our trauma unit following a motor vehicle accident. He was the driver (unrestrained) of a mini-vehicle traveling at high speed, when it collided with a power post. The vehicle 
sustained only moderate damage, and at the scene, ambulance staff reported that the patient appeared to be well. He was brought into our unit for further assessment.

On admission the patient appeared well. He was alert, orientated and had only minor abrasions to the forehead. His glasgow coma scale was 15 and he had full recollection of the event. His airway was patent, with non-tender cervical spine. His respiratory rate was $14 / \mathrm{min}$, heart rate was $90 / \mathrm{min}$ and blood pressure was $135 / 65 \mathrm{mmHg}$. The patient reported no specific complaints, but it was noted that he had a slight and ill defined, generalized tenderness in his abdomen. He had no hematuria and his emergency bedside ultrasound was negative. At this time, his hemoglobin was $12.5 \mathrm{~g} / \mathrm{dL}$ and serum lactate was 1 . Cervical spine, chest and pelvic radiographs were unremarkable.

The patient was given analgesia, and over the next four hours he was reviewed by the duty junior trauma resident (year one resident, in the first month of the rotation), by which time the patient's abdominal discomfort had almost subsided. The patient remained hemodynamically stable, and he had no further complaints. However, based on the mechanism of injury, and the concern of the possibility of occult intraabdominal organ injury, an urgent double contrast (oral and intravenous) done CT scan of the abdomen was (64 slice multi-detector). The scan was reviewed by the duty radiologist and was reported as normal. He was eventually discharged from the unit 24 hours from the time of his initial injury.

Unfortunately, 48 hours after being discharged, the patient represented with nausea, vomiting and a progressively increasing amount of abdominal pain. On arrival, he was in distress, and was diaphoretic, with a respiratory rate $40 / \mathrm{min}$, heart rate $120 / \mathrm{min}$, and blood pressure $125 / 70 \mathrm{mmHg}$. His abdomen was grossly distended (Figure 1), and he had generalized peritonitis. His hemoglobin $11 \mathrm{~g} / \mathrm{dL}$, and serum lactate was 7.5. An erect chest radiograph showed no pneumoperitonium.

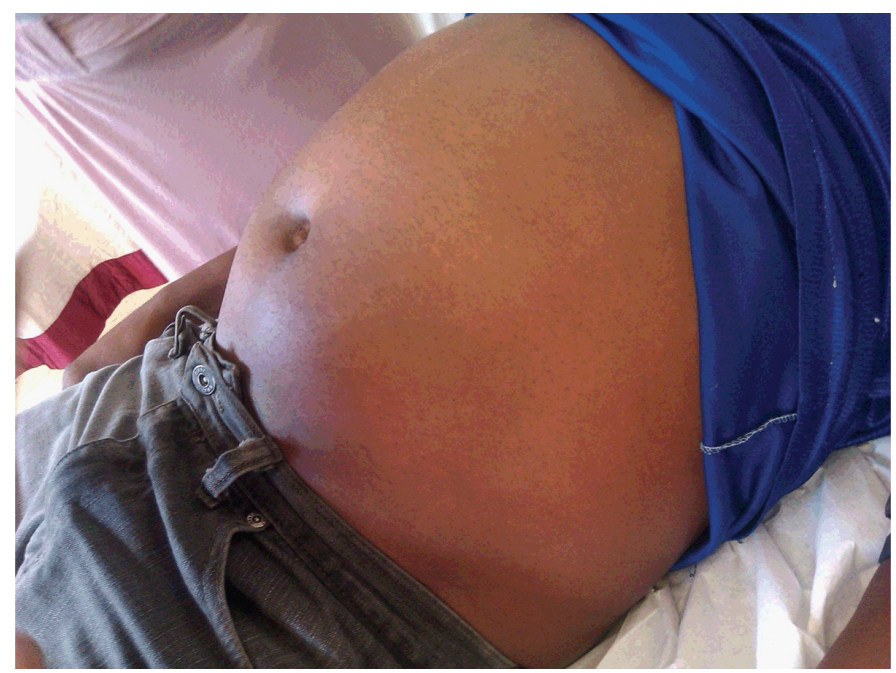

Figure 1: Marked abdominal distension 48 hours after discharge from the trauma unit.
The original CT scan was reviewed by two senior radiologists, both of whom reported no abnormalities.

An emergency laparotomy was performed. Approximately $500 \mathrm{~mL}$ of clotted blood was noted on entry into the abdominal cavity. A massive small bowel mesenteric defect, with no active hemorrhage was found (Figure 2). A 20-cm segment of devascularized, dilated and necrotic small bowel was noted $(100 \mathrm{~cm}$ from the duodenal jejunal flexure), with evidence of imminent perforation (Figure 3). There was no other injury. This necrotic portion was resected, and an end-to-end anastomosis was performed. He remained stable intraoperatively and was transferred to the general trauma ward. He had an uneventful recovery and was discharged on day- 6 .

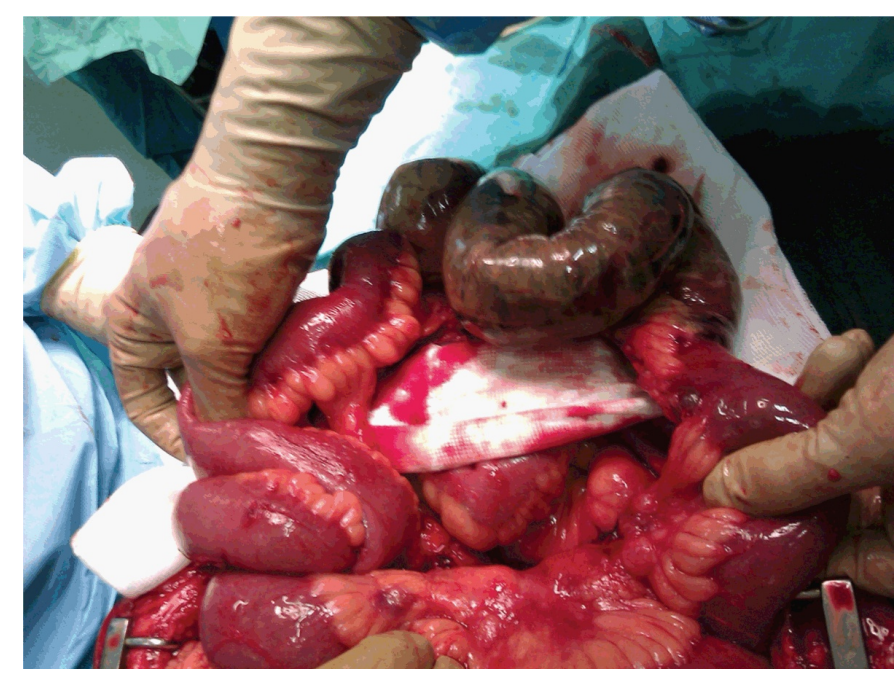

Figure 2: Large mesenteric defect, with devascularized small bowel.

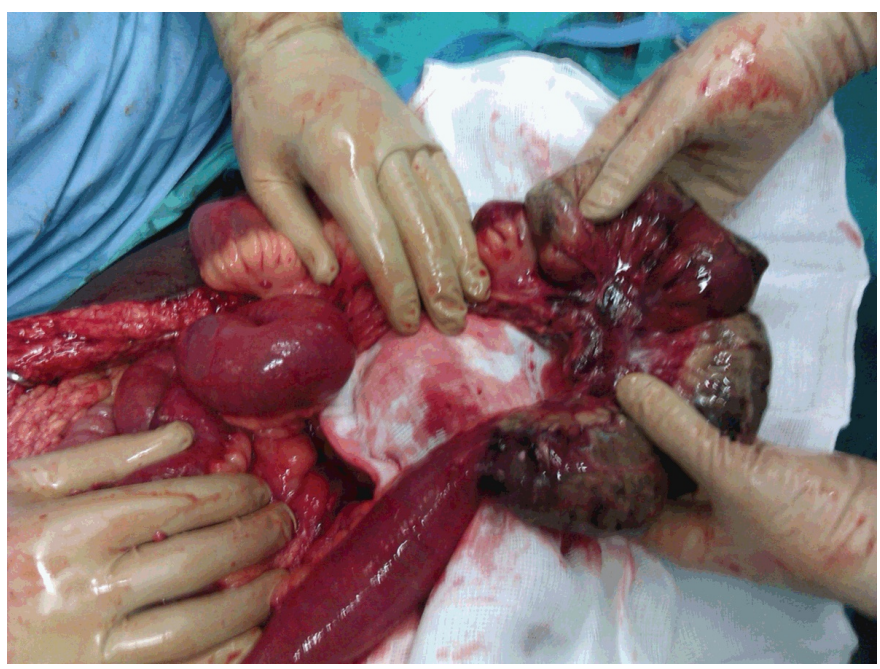

Figure 3: Infarcted small bowel segment with evidence of imminent perforation. 


\section{DISCUSSION}

Mesenteric injury following blunt abdominal trauma is uncommon and has a reported incidence of approximately $1 \%[1-3]$. One of the most common mechanisms involves rapid deceleration, which produces a shearing force between fixed and mobile portions of the intestinal tract, leading to mesenteric disruption [4].

This case highlights the difficulty of diagnozing mesenteric injury in blunt abdominal trauma patients as clinical presentation is often extremely subtle. The history and physical examination may be unreliable as most patients have either minimal physical signs, or concurrent distracting injuries. The 'classic' clinical triad of abdominal pain, rigidity and absent bowel sounds is only seen in less than one-third of all patients [5]. The delayed diagnosis of mesenteric injury is known to be associated with significant morbidity and even mortality [6].

Multi detector computed tomography (MDCT) is currently the gold standard in evaluating blunt abdominal trauma in hemodynamically stable patients [3]. However, studies evaluating the diagnostic accuracy of CT in identifying mesenteric injuries have been conflicting. Earlier studies reporting relatively lower rate of correct identification of mesenteric injuries largely predated MDCT. Currently, evidence from multiple recent studies reported better diagnostic accuracy with a sensitivity ranging from $69-95 \%$ and a specificity of $94-100 \%$ [7].

Numerous radiological signs have been described in literature, but many are non-specific [8]. One of the key objectives in interpreting the CT findings in these patients is to establish the presence of significant mesenteric injuries that may require surgical intervention. However, in spite of the wide availability of CT as part of routine trauma evaluation in these patients, there is likely to remain a proportion of patients in whom injuries can inevitably be missed. [8].

Patients who are hemodynamically stable with negative findings on CT are often admitted for repeat clinical evaluation. However, the incidence of significant intra-abdominal injuries in these patients is relatively low, and this has been reported to be less than $1 \%$ of cases. [9]. Although protocols and the duration of observation vary between units, some have argued that clinical observations longer than 24 hours solely to attempt identifying the small proportion of missed injuries may not be justified. A repeat CT scan in selected patients have been advocated by some authors, but is only useful in patients with concurrent head and other multiple injuries [10].

Interestingly, this patient presented with minimal abdominal pain on initial presentation, and remained unchanged within the first 24 hours after the injury. This was possibly due to early presentation of disruption of the mesentery with initial devascularization of the short bowel segment, which produced minimal physical findings. In our patient, the symptoms of ischemic bowel with imminent perforation as a sequalae of undiagnosed mesenteric injury only presented after 24 hours from the time of injury. The possibility of occult intra-abdominal injury was correctly entertained by the duty junior trauma resident, leading to the use of CT scan for its evaluation. The normal CT scan in this case gave an erroneous reassurance that no significant injury was sustained.

Clinical decision making can be challenging, especially for the junior residents at the 'front-line', who are often overwhelmed by the massive volume of major trauma seen. Furthermore, due to severe pressure for in patient beds (as is often the case in major trauma center in the developing world) it may not always be possible to admit every patient for extended period of observation, even if it may be justified on clinical grounds.

Based on the severity of the mechanism of injury and the recognition of high-risk for potential injuries, there is a need to remain vigilent and consider extending the observation period beyond 24 hours, even if patients appeared 'well' initially. The policy of selective admission, however, must be applied judiciously in highly selected patients. Currently, without accurate predictors to aid patient selection, this decision remains difficult, especially for the inexperienced.

\section{CONCLUSION}

Mesenteric injury following significant abdominal trauma is rare but carries significant morbidity as delay in recognition is common due to subtle clinical presentation. Although computed tomography (CT) scan is reasonably accurate in identifying such injury, it still cannot be completely excluded by a negative scan and particularly if the computed tomography (CT) scan is carried out within a very short time frame post blunt abdominal trauma. Clinicians must always remain vigilant of the possibility of mesenteric injury and not be falsely reassured by apparently normal radiological findings. Extending clinical observation periods for selected patients, based on mechanism of injury and ongoing clinical suspicion, is advisable in order to minimize the chances of missing clinically significant mesenteric trauma and resultant devascularization of small bowel.

$* * * * * * * * *$

\section{Author Contributions}

Victor Kong - Substantial contributions to conception and design, Acquisition of data, Analysis and interpretation of data, Drafting the article, Revising it critically for important intellectual content, Final approval of the version to be published

John Burce - Acquisition of data, Analysis and interpretation of data, Drafting the article, Revising it critically for important intellectual content, Final approval of the version to be published

George Oosthuizen - Analysis and interpretation of data, Drafting the article, Revising it critically for 
important intellectual content, Final approval of the version to be published

Grant Laing - Analysis and interpretation of data, Drafting the article, Revising it critically for important intellectual content, Final approval of the version to be published

Damian Clarke - Analysis and interpretation of data, Drafting the article, Revising it critically for important intellectual content, Final approval of the version to be published

\section{Guarantor}

The corresponding author is the guarantor of submission.

\section{Conflict of Interest}

Authors declare no conflict of interest.

\section{Copyright}

(C) Victor Kong et al. 2013; This article is distributed under the terms of Creative Commons Attribution 3.0 License which permits unrestricted use, distribution and reproduction in any means provided the original authors and original publisher are properly credited. (Please see www.ijcasereportsandimages.com /copyright-policy.php for more information.)

\section{REFERENCES}

1. Watts DD, Fakhry SM. Incidence of hollow viscus injury in blunt trauma: an analysis from 275, 557 trauma admissions from the East multi-institutional trial. J Trauma 2003;54(2):289-4.
2. Imtiaz Wani, Rayees A Bhat, Shayiq Wani, Nawab Khan, Rauf A Wani, Fazal Q Parray. Isolated Small Bowel Mesentery Injury After Steering Wheel Trauma. Trauma Mon 2012;17(2):279-81.

3. Killeen KL, Shanmuganathan K, Poletti PA, Cooper C, Mirvis SE. Helical computed tomography of bowel and mesenteric injuries. J Trauma 2001 Jul;51(1):26-36.

4. Hughes TM, Elton C. The pathophysiology and management of bowel and mesenteric injuries due to blunt trauma. Injury 2002;33(4):295-302.

5. Levine CD, Gonzales RN, Wachsberg RH, Ghanekar D. CT findings of bowel and mesenteric injury. $\mathrm{J}$ Comp Assist Tomogr 1997;21(6):974-9.

6. Scaglione M, de Lutio di Castelguidone E, Scialpi M, et al. Blunt trauma to the gastrointestinal tract and mesentery: is there a role for helical CT in the decision- making process? Eur J Radiol 2004;50(1):67-3.

7. Brofman N, Atri M, Hanson JM, Grinblat L, Chughtai T, Brenneman F. Evaluation of bowel and mesenteric blunt trauma with multidetector CT. Radiographics 2006;26(4):1119-31.

8. Ekeh AP, Saxe J, Walusimbi M, et al. Diagnosis of blunt intestinal and mesenteric injury in the era of multidetector CT technology--are results better? J Trauma 2008;65(2):354-9.

9. Kendall JL, Kestler AM, Whitaker KT, Adkisson MM, Haukoos JS. Blunt Abdominal Trauma Patients Are at Very Low Risk for Intra-Abdominal Injury after Emergency Department Observation. West J Emerg Med 2011;12(4):496-504.

10. Stephan PJ, McCarley MC, O'Keefe GE, Minei JP. 23-Hour observation solely for identification of missed injuries after trauma: is it justified? J Trauma 2002;53(5):895-900.
Access full text article on other devices

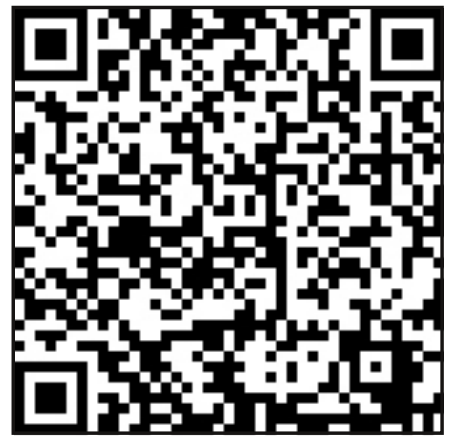

Access PDF of article on other devices

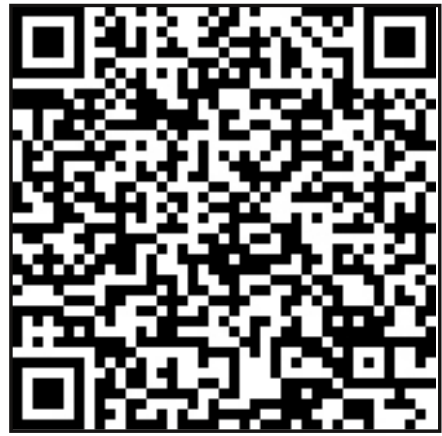

